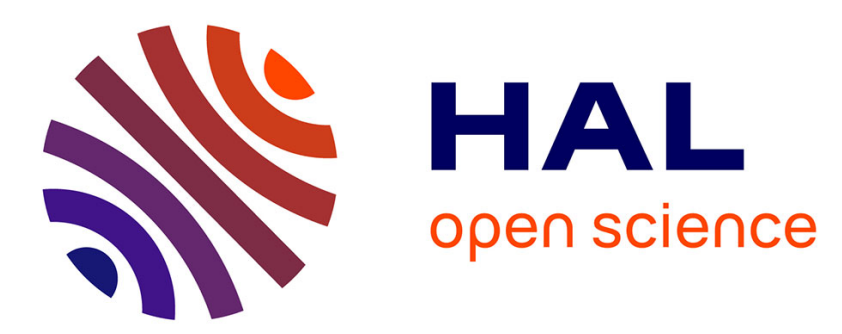

\title{
A Numerical Model for Powder Densification by SPS Technique
}

\author{
Pamela Mondalek, Luisa Silva, Michel Bellet
}

\section{To cite this version:}

Pamela Mondalek, Luisa Silva, Michel Bellet. A Numerical Model for Powder Densification by SPS Technique. Advanced Engineering Materials, 2011, 13 (7), pp.587-593. 10.1002/adem.201000340 . hal-00603968

\section{HAL Id: hal-00603968 \\ https://hal-mines-paristech.archives-ouvertes.fr/hal-00603968}

Submitted on 25 Apr 2014

HAL is a multi-disciplinary open access archive for the deposit and dissemination of scientific research documents, whether they are published or not. The documents may come from teaching and research institutions in France or abroad, or from public or private research centers.
L'archive ouverte pluridisciplinaire HAL, est destinée au dépôt et à la diffusion de documents scientifiques de niveau recherche, publiés ou non, émanant des établissements d'enseignement et de recherche français ou étrangers, des laboratoires publics ou privés. 
A numerical model for powder densification by SPS technique**

By P. Mondalek*, L. Silva and M. Bellet

[*] P. Mondalek, Dr. L. Silva, Prof. M. Bellet

MINES ParisTech, Centre de Mise en Forme des Matériaux (CEMEF),

UMR CNRS 7635, 06904 Sophia-Antipolis, France

E-mail: pamela.mondalek@mines-paristech.fr

[**] This study has been conducted in the framework of the cooperative project "IRIS" supported by the French Agence Nationale de la Recherche (ANR).

Spark Plasma Sintering belongs to a class of sintering techniques that employs electric current to assist compaction. This technology seems very promising to obtain net-shape components made of intermetallic alloys with a fine microstructure. However the SPS process is difficult to stabilize because of density heterogeneities arising from non homogeneous temperature or stress in the powder. This motivates the development of a three-dimensional finite element simulation in order to understand the distribution of current, temperature and porosity. The model couples three physical problems: electrical, thermal and mechanical. The numerical implementation is based on a monolithic formulation consisting in solving the different conservation equations on a single mesh including the specimen and the tooling. The general set of equations is described; the effect of the powder physical properties and of the geometry of the set up on the distribution of electrical current and temperature is discussed. A macroscopic Abouaf constitutive model is used to simulate powder densification; first results involving porosity evolution are presented.

Powder sintering technique assisted by electrical current, which is also called Spark Plasma Sintering (SPS), is a revolutionary high speed powder consolidation technology. This process presents many benefits such as reduction of sintering times and capacity of producing 
materials with excellent properties. In fact, the speed of the process allows powder densification with nanostructured materials. The SPS is used to produce different materials like ceramics, composites, metals and also intermetallic alloys that are difficult to form with conventional techniques due to their different melting temperatures. ${ }^{[1]}$ Application of SPS to high performance intermetallics should allow their cost-effective introduction in aero-engines, in which these low density materials have great potential to improve fuel efficiency and reduce the amount of pollution. ${ }^{[2]}$ The main characteristic of SPS is that heat is generated internally by Joule effect, in contrast to conventional hot pressing, where heat is provided by conduction and/or radiation. More precisely, the process consists in applying a pulsed continuous current and a uniaxial pressure to obtain nanostructured materials due to very high heating rates and short sintering time (about $15 \mathrm{~min}$ ). The powder material undergoes different mechanisms leading to full compaction, such as plastic deformation, sintering electromigration and surface diffusion induced by surface tension. During compaction, the particle size and current porosity value influence those different contributions. ${ }^{[3-4]}$ In addition, there is a strong influence of temperature which is directly connected to the distribution of current. $^{[5]}$ Therefore, to control the microstructure homogeneity, especially for complex components, it is important to understand current flow, heat transfer, densification mechanisms and how they are coupled. A 3D numerical model has been developed using the CimLib library (implemented at CEMEF) to simulate the coupled electrical thermal mechanical problems. The finite element modeling and the equations are presented in the first section. The monolithic approach, on which the numerical code is based, is then presented. Finally, results concerning the distribution of temperature and density are discussed.

\section{Finite element modeling}

Governing equations: coupled problems 
The finite element modeling of the SPS process is based on a set of governing equations. ${ }^{[6-}$ ${ }^{7-8]}$ The powder is placed inside a graphite mold and is surrounded by two graphite pistons on the top and bottom. An electrical current is then applied. Since all the tools are conductive, the electric current goes through the whole assembly and generates heat. Simultaneously, loads are applied to assist sintering. As a consequence, a compaction of the powder density is observed. Therefore, due to the interaction between the electric current, temperature and densification behaviour, three coupled problems need to be solved: electrical, thermal and mechanical.

First, the electrical modeling in the assembly (powder and tooling) is based on the charge conservation law:

$$
\nabla \cdot \overrightarrow{\mathbf{J}}=0
$$

Applying Ohm's law, the current density $\overrightarrow{\mathbf{J}}$ can be written:

$$
\overrightarrow{\mathbf{J}}=\sigma_{e} \overrightarrow{\mathbf{E}}=-\sigma_{e} \nabla U
$$

where $\overrightarrow{\mathbf{E}}=-\nabla U$ is the electric field and $\sigma_{e}$ the electric conductivity. The solution of the electrical problem consists in solving the following Poisson type equation for the electrical potential $U$ :

$$
\nabla \cdot\left(-\sigma_{e} \nabla U\right)=0
$$

As a consequence of electric flow, heat is generated in the assembly by Joule effect. The associated source term in the heat equation is written: $q_{e}=\overrightarrow{\mathbf{J}} \cdot \overrightarrow{\mathbf{E}}$. The temperature distribution is calculated by solving the heat equation:

$$
\nabla \cdot(-k \nabla T)+\rho c_{p} \frac{d T}{d t}=q_{e}
$$

where $T$ is the temperature, $k$ is the thermal conductivity, $\rho$ the density, $c_{p}$ the specific heat and $q_{e}$ the heat source. In this first approach, these parameters do not depend on the porosity. 
Furthermore, the powder is subjected to mechanical loads exerted by the pistons. Stresses are generated inside the powder and tools. Here, as a first approach, tooling components are considered rigid. The mechanical problem in the powder (considered here as a continuum) is governed by the momentum equation, in which gravity and inertia effects are ignored:

$$
\nabla \cdot \boldsymbol{\sigma}=0
$$

where $\sigma$ is the stress tensor in the powder.

The densification of the powder during electrically activated sintering results from different physical phenomena: ${ }^{[3]}$ plastic deformation of grains, mass transport between grains by diffusion under load, electro-migration, or by surface tension. Therefore, the global strain rate of the compressible continuum is the sum of four contributions:

$$
\dot{\boldsymbol{\varepsilon}}=\dot{\boldsymbol{\varepsilon}}^{p l}+\dot{\boldsymbol{\varepsilon}}^{d l}+\dot{\boldsymbol{\varepsilon}}^{e m}+\dot{\boldsymbol{\varepsilon}}^{s t}
$$

It is assumed here that plastic deformation of powder particles is the dominant phenomenon and consequently, in a first approach, only the contribution $\dot{\boldsymbol{\varepsilon}}^{p l}$ is considered. A macroscopic model is used to follow the powder density evolution. More precisely, the powder is considered as a continuum characterized by the rate of its relative density, which is defined as the ratio of the material volume by the apparent volume $\left(\rho_{r}=V_{m} / V_{a}\right)$. The density of the continuum is then expressed as $\rho=\rho_{r} \rho_{0}$, where $\rho_{0}$ the density in the dense state. The framework is the same as the one proposed by Shima \& Oyane complemented by the thermo dynamical justifications of Abouaf et al.. ${ }^{[9-10]}$ For a given metallic alloy, supposing that the dense material obeys a one-dimensional constitutive equation $\dot{\bar{\varepsilon}}=\mathrm{F}(\bar{\sigma}, T)$, where $\bar{\sigma}$ and $\dot{\bar{\varepsilon}}$ denote respectively the von Mises generalized stress and the generalized plastic strain rate, then the powder continuum composed of particles of the same alloy satisfies the same relation, but with the following expressions for invariants $\bar{\sigma}$ and $\dot{\bar{\varepsilon}}$, proposed by Green: ${ }^{[1]}$

$$
\bar{\sigma}=\left[\frac{3}{2} c\left(\rho_{r}\right) \operatorname{dev}(\boldsymbol{\sigma}): \operatorname{dev}(\boldsymbol{\sigma})+f\left(\rho_{r}\right)(\operatorname{tr} \boldsymbol{\sigma})^{2}\right]^{1 / 2}
$$




$$
\dot{\bar{\varepsilon}}=\left[\frac{2}{3 c\left(\rho_{r}\right)} \dot{\boldsymbol{\varepsilon}}: \dot{\boldsymbol{\varepsilon}}+\left(\frac{1}{9 f\left(\rho_{r}\right)}-\frac{2}{9 c\left(\rho_{r}\right)}\right)(\operatorname{tr} \dot{\boldsymbol{\varepsilon}})^{2}\right]^{1 / 2}
$$

Parameters $c$ and $f$ are two decreasing functions of the relative density $\rho_{r}$. In fact, $c$ and $f$ get their minimum value, 1 and 0 respectively, when densification is completed. During SPS compaction, high temperatures are reached and maintained for a few minutes (typically $800^{\circ} \mathrm{C}$ to $1200^{\circ} \mathrm{C}$, in 10 to $20 \mathrm{~min}$ ), so a viscoplastic model is considered based on a Norton-Hoff formulation. The viscoplastic potential $\varphi$ is written:

$$
\varphi=\frac{m}{m+1} K(T)\left(\frac{\bar{\sigma}}{K \sqrt{3}}\right)^{\frac{m+1}{m}}
$$

where $K(T)$ is the material consistency and $m$ the strain rate sensitivity index. According to Abouaf, ${ }^{[10]} \dot{\bar{\varepsilon}}$ in the porous state should be expressed as in the dense state:

$$
\dot{\bar{\varepsilon}}=\frac{\partial \varphi}{\partial \bar{\sigma}}
$$

Consequently the stress tensor can be separated into deviatoric and volumetric contributions and we write respectively the deviatoric stress tensor and the hydrostatic pressure as:

$$
\begin{gathered}
\mathbf{s}=\frac{2 K(T)}{c\left(\rho_{r}\right)}(\sqrt{3} \dot{\bar{\varepsilon}}(\mathbf{v}))^{m-1} \dot{\mathbf{e}}(\mathbf{v}) \quad \text { with } \quad \dot{\mathbf{e}}(\mathbf{v})=\operatorname{dev}\left(\dot{\boldsymbol{\varepsilon}}^{v p}(\mathbf{v})\right) \\
p=-\frac{1}{3} \operatorname{tr}(\boldsymbol{\sigma})=-K(T)(\sqrt{3} \dot{\bar{\varepsilon}}(\mathbf{v}))^{m-1} \frac{1}{3 f\left(\rho_{r}\right)} \operatorname{tr}\left(\dot{\boldsymbol{\varepsilon}}^{v p}(\mathbf{v})\right)
\end{gathered}
$$

In this study, and as a first approach, friction between powder and tooling is neglected, perfect slip conditions are considered.

The mechanical problem includes another equation from which the relative density is deduced. More precisely, during compaction, closing of pores causes the variation of the apparent volume and so the relative density. Since $\rho=\rho_{r} \rho_{0}$, the evolution of $\rho_{r}$ is calculated by solving mass conservation equation:

$$
\frac{\partial \rho_{r}}{\partial t}+\nabla \rho_{r} \cdot \mathbf{v}+\rho_{r} \nabla \cdot \mathbf{v}=0
$$

where $\mathbf{v}$ denotes the velocity field of the powder continuum. Once calculated, $f$ and $c$ functions are updated using the following expressions: ${ }^{[12]}$ 


$$
f=k_{f} \frac{1-\rho_{r}}{\rho_{r}-\rho_{c}} \quad \text { and } \quad c=\alpha_{c} f+1
$$

Where $k_{f}, \rho_{c}$ and $\alpha_{c}$ are material parameters. All physical properties are dependent on the temperature and are implemented as functions of the temperature calculated at each time step.

\section{Numerical approach}

The electric and thermal problems are solved using a monolithic method, through the CimLib library developed at CEMEF. The assembly is represented by a single mesh (Fig. 1) and a single equation is solved for each problem on the entire geometry. As a result, the different boundary conditions between the different components of the SPS assembly, like heat conduction, are avoided but on the other hand, specific methods are used in order to characterize the subdomains occupied by the different materials. The concept behind is based on using a function to differentiate the materials and on a mesh adaptation technique providing mesh control at the interfaces.

Let $\Omega$ be the computational domain, divided into a certain number of subdomains $\Omega_{\mathrm{i}}$ where each subdomain refers to the domain occupied by the powder or by the different materials constituting the parts of the assembly: dies, spacers and pistons. These subdomains $\Omega_{\mathrm{i}}$ are described with level set functions $\alpha_{\mathrm{i}}(\mathrm{x})$, each one being the signed distance to the interface $\Gamma_{\mathrm{i}}$ boundary of $\Omega_{\mathrm{i}} \cdot{ }^{[13]}$ Therefore, the function $\alpha_{\mathrm{i}}(\mathrm{x})$ is expressed as follows:

$$
\alpha_{i}(\mathbf{x})= \begin{cases}\mathrm{d}\left(\mathbf{x}, \Gamma_{i}\right) & \text { if } \mathbf{x} \in \Omega_{i} \\ -\mathrm{d}\left(\mathbf{x}, \Gamma_{i}\right) & \text { if } \mathbf{x} \notin \Omega_{i} \\ 0 & \text { if } \mathbf{x} \in \Gamma_{i}\end{cases}
$$

Once calculated, the level set allows us to define a presence function of the subset $\Omega_{\mathrm{i}}$. A "smoothed" Heaviside function $\mathrm{H}\left(\alpha_{\mathrm{i}}\right)$ is used in this work, where a thickness $\varepsilon_{m}$ is fixed in the surrounding of the interface in order to prevent the discontinuous transition in the region: 


$$
\mathrm{H}\left(\alpha_{i}\right)=\left\{\begin{array}{lll}
0 & \text { if } & \alpha_{i}<-\boldsymbol{\varepsilon}_{m} \\
\frac{1}{2}\left(1+\frac{\alpha_{i}}{\boldsymbol{\varepsilon}_{m}}+\frac{1}{\pi} \sin \left(\frac{\pi \alpha_{i}}{\boldsymbol{\varepsilon}_{m}}\right)\right) & \text { if } & \left|\alpha_{i}\right| \leq \boldsymbol{\varepsilon}_{m} \\
1 & \text { if } & \alpha_{i}>\boldsymbol{\varepsilon}_{m}
\end{array}\right.
$$

In calculations, the value of $\varepsilon_{m}$ is chosen accordingly to the mesh size. Furthermore, the objective being to calculate the physical properties on the entire geometry, mixing laws are introduced to express the properties on $\Omega$. These laws are defined as functions of the level set. Different expressions can be used, either approximating at nodes or at elements $(\mathrm{Pl}$ or $\mathrm{PO}$ type). As an example, for the density and specific heat, linear interpolations are applied at the neighborhood of the interface between the two subdomains $i$ and $j$ :

$$
\rho=\mathrm{H}\left(\alpha_{i}\right) \rho_{i}+\left(1-\mathrm{H}\left(\alpha_{i}\right)\right) \rho_{j} \quad c_{p}=\mathrm{H}\left(\alpha_{i}\right) c_{p_{i}}+\left(1-\mathrm{H}\left(\alpha_{i}\right)\right) c_{p_{j}}
$$

Besides, same approach is used for the conductivities; but it can be shown that harmonic average provides better results:

$$
\frac{1}{\sigma_{e}}=\frac{\mathrm{H}\left(\alpha_{i}\right)}{\sigma_{e, i}}+\frac{\left(1-\mathrm{H}\left(\alpha_{i}\right)\right)}{\sigma_{e, j}} \quad \frac{1}{k}=\frac{\mathrm{H}\left(\alpha_{i}\right)}{k_{i}}+\frac{\left(1-\mathrm{H}\left(\alpha_{i}\right)\right)}{k_{j}}
$$

Whereas, concerning the consistency $K$ and the strain rate sensivity coefficient $m$, discontinuous mixing laws show better results, following expressions are used:

$$
\psi_{\Omega_{e}}=\frac{\psi_{i}\left|\Omega_{i} \cap \Omega_{e}\right|+\psi_{j}\left|\Omega_{j} \cap \Omega_{e}\right|}{\left|\Omega_{e}\right|}
$$

$\Omega_{\mathrm{e}}$ being the element on which the mixing is applied, $\psi_{i}$ and $\psi_{j}$ the respective properties of $\Omega_{i}$ and $\Omega_{j}$.

In order to enhance the accuracy around the interface, the level set method and mixing laws are associated with an anisotropic adaptive meshing. More precisely, the main point is to keep an isotropic mesh in the area far from the interface, and to generate a refined mesh with anisotropic elements in its neighbourhood. For that reason, the metric used for the mesh is dependent on the gradient of the level set function: 


$$
\mathbf{M}=\left\{\begin{array}{ll}
\varepsilon_{r e m} \mathbf{I} & \text { if }|\alpha|>e \\
\left(\frac{N}{2 e}-\varepsilon_{r e m}\right) \mathbf{A}+\varepsilon_{r e m} \mathbf{I} & \text { otherwise }
\end{array} \quad \text { where } \quad \mathbf{A}=\frac{\nabla \alpha \otimes \nabla \alpha^{T}}{\|\nabla \alpha\|^{2}}\right.
$$

where $N$ is the number of elements generated in the thickness $2 e$ and in the direction of the gradient of the level set, and $\varepsilon_{r e m}$ is related to the background mesh size. In practice, the mesh is generated using the MTC mesher developed by Coupez. ${ }^{[14-15]}$

\section{Simulations}

Figure 1 presents the 3D geometry considered in the simulation of the electric-thermalmechanical coupled problems. Due to symmetry reasons, only a quarter of the set up is simulated.

An electrical current density is applied on the upper graphite edge and a constant zero potential is applied on the lower graphite edge. The current density is continuously controlled by a PID algorithm ("Proportional-Integral-Derivative"). The temperature is controlled in the mould $3 \mathrm{~mm}$ away from the specimen surface. At each time step, the injected current density $J$ is calculated as a function of the error between calculated and prescribed temperature if $K_{p}$, $K_{i}$ and $K_{d}$ are the proportional, integral and derivative constants $J$ is written:

$$
J^{t}=K_{p} \times \text { error }^{t}+K_{i} \sum_{t=0}^{t} \text { error }^{t}+K_{d} \times\left(\text { error }^{t}-\text { error }^{t-1}\right) \quad \text { error }=T_{\text {prescribed }}-T_{\text {calc }}
$$

Two different material samples are chosen: $\mathrm{TiAl}$ and $\mathrm{Al}_{2} \mathrm{O}_{3}$. The mould, pistons and spacers are made of graphite. To minimize heating rates in the SPS setup, two Inconel spacers are in contact with the graphite edges and are water cooled (Fig. 1). As Inconel pieces are not included in our numerical domain, it is necessary to express the thermal boundary condition directly onto the boundaries of the top and bottom graphite spacers. This can be done as follows. Assuming a quasi-stationary state, the heat flux at the Inconel boundary is equal to the heat flux through the Inconel piece, and to the heat flux through the interface between Inconel and graphite. 


$$
\phi=h_{\text {cond }}\left(T_{\text {inconel edge }}-T_{\text {water }}\right)=k_{\text {inconel }} \frac{T_{\text {inconel } \cap \text { graphite }}-T_{\text {inconeledge }}}{e_{\text {inconel }}}
$$

As a consequence, using these two expressions, the heat flux on the graphite boundary can be expressed as a function of the graphite temperature along this boundary and the water temperature:

$$
\phi=h_{\text {cond }} \frac{k_{\text {inconel }} / e_{\text {inconel }}}{h_{\text {cond }}+k_{\text {inconel }} / e_{\text {inconel }}}\left(T_{\text {graphite }}-T_{\text {water }}\right)
$$

here $h_{\text {cond }}=880 \mathrm{~W} \cdot \mathrm{m}^{-2} \cdot \mathrm{K}^{-1}$ is a constant heat transfer coefficient, $k_{\text {inconel }}$ the Inconel thermal conductivity, $e_{\text {inconel }}$ its thickness and $T_{\text {water }}$ the water temperature (assumed constant $296 \mathrm{~K}$ ). Moreover, the process taking place in a vacuum chamber, heat losses by convection and conduction are neglected. All lateral surfaces have heat losses by radiation towards chamber walls, which are held at room temperature $(300 \mathrm{~K})$, a radiative heat flux is considered:

$$
\phi_{\text {radiation }}=\sigma_{B} \varepsilon_{r}\left(T^{4}-T_{\text {wall }}^{4}\right)
$$

where $\sigma_{\mathrm{B}}=5.67 \times 10^{-8} \mathrm{~W} \cdot \mathrm{m}^{-2} \cdot \mathrm{K}^{-4}$ is the Stefan-Boltzmann's constant, $\varepsilon_{r}$ the emissivity (assumed to be equal to 0.8 in this study), $T$ the local temperature of the vertical boundary surfaces and $T_{\text {wall }}$ the temperature of the chamber walls. On the other hand, a constant voltage is applied during the simulations. Furthermore, all contact surfaces between the parts of the SPS assembly are considered ideal: no contact resistance is taken into account. Physical parameters are taken from Molenat et al.. ${ }^{[8]}$

The powder follows the viscoplastic law for porous materials as mentioned previously. Besides, tools are assumed to be rigid (stresses are not calculated inside graphite) and a frictionless contact is considered between tools and powder. Moreover, a constant velocity of $6 \times 10^{-3} \mathrm{~mm} / \mathrm{s}$ is applied to the upper tooling, whereas the mould and lower tooling are considered fixed. The initial porosity in the TiAl powder is considered to be equal to $30 \%$. The consistency $K(T)$ and the strain rate sensitivity $m(T)$ are thermal dependent, their values are deduced from literature: measurements carried out on $\mathrm{Ti}-47 \mathrm{Al}-2 \mathrm{Cr}-2 \mathrm{Nb} .^{[16]}$ 


\section{Results}

\section{Electrical potential and temperature distribution}

The distribution of the temperature during the SPS process is directly related to the distribution of current which is firstly affected by the geometry and the physical properties of the different components and materials involved in the whole set-up. The choice of the two samples, with two different electrical conductivities, shows their effect on the distribution of the current and consequently on the distribution of the temperature. In Fig. 2, the norm of the current density is compared. Results show that the highest current density is in the region of the pistons in both cases, due to their smaller section. Besides, in the case of TiAl, most of the current flows through the sample, the distribution of the current density is more important at the lateral surface of the conducting specimen and decreases in the radial direction towards the centre during the process. Whereas in the case of alumina the current is, as expected, relatively absent inside the sample, because of its low electrical conductivity at low temperature. In fact, at the beginning, alumina is heated only by conduction and not directly by Joule effect, its electrical conductivity increases with temperature, allowing the current to go through after $15 \mathrm{~min}$, as it can be seen in figure 2 .

Since the homogeneity of the microstructure in the SPS parts is directly dependent on the temperature homogeneity, it is interesting to understand the evolution of the temperature distribution during the process. This was studied in several works. ${ }^{[5-6]}$ Pistons are heated first due to the concentration of electrical current in their reduced section and present the highest temperatures along the process, that way increasing heating of the powder by conduction. Besides, the gradient of temperature inside the sample is directly related to the electrical conductivity since the current is the only heat source. ${ }^{[6]}$ Actually, the electrical conductivity of the powder affects the distribution of the current in the graphite that surrounds it (Fig. 2), consequently the distribution of the current in graphite affects the distribution of the source 
term and so the temperature gradient inside the sample. As shown in the graphics of figure $\mathbf{3}$, the temperature gradient inside the alumina specimen varies with time. The increase of its electrical conductivity with temperature changes the current distribution and so the heating phenomenon.

Moreover, geometry of the setup could be a major cause of heterogeneities. When the load is applied, the symmetry in the vertical direction might be lost. Coupling with the mechanical problem allows controlling this source of homogeneity. This can be seen in figure 4 , where a displacement is imposed in the upper tools to compact the powder while the lower tools are kept fixed.

In figure 5, the axial temperature profiles after $25 \mathrm{~s}$ and $900 \mathrm{~s}$ show that once symmetry is lost, heating is no longer similar on both sides. When the piston enters into the mould, the height of the restricted section is lower. Consequently, Joule effect is less important since the concentration of the current flux covers a smaller region.

The temperature heterogeneities in the sample affect directly the mechanical problem. Since the constitutive law is temperature dependant, the deformation resulting from the pressure applied during the SPS process will be heterogeneous. Densification won't be achieved identically inside the specimen. In order to better display the evolution of porosity, a different geometry is chosen to simulate the mechanical problem (figure 6). Same boundary conditions are used as previously. The fully coupled algorithm (electrical, thermal and mechanical) is used. Then all level set functions are updated as well as remeshing to conserve interfaces. The norm of the current density is presented in Fig. 6, the current is concentrated in the bottom of the powder where the radius is smaller. However, the source term of the heat equation is not affected significantly and so the gap of temperature in the powder doesn't exceed $40{ }^{\circ} \mathrm{C}$. 
In that case, pressure gradients result mainly from the geometry. The pressure is the highest in the upper section of the specimen and decreases towards the centre and the bottom. Since the consistency decreases with temperature, the pressure decreases with time as seen in figure 7. The heterogeneity of pressure induces the heterogeneity during densification; the porosity is lower when pressure is high. The initial relative density is set to 0.7 , after 16 min it reaches 0.99 in the upper region where pressure is the highest, as seen in figure $\mathbf{8}$.

Hence, simulations allow predicting densification during the SPS process in order to optimize the compacted specimen. The position of the specimen in the mould and its orientation is one of the major issues that affect homogeneity.

\section{Conclusions}

A 3D finite element analysis has been presented to simulate the electrical thermal and mechanical problems during the SPS process. It is based on CimLib using a monolithic approach. Its application to two samples (TiAl and alumina) has proven that distribution of current strongly depends on geometry and physical properties. It was shown how, in the case of alumina, the increase of its electrical conductivity causes a variation of the temperature distribution inside the powder. Besides by coupling with the mechanical problem, the distribution of the temperature in the axial direction was shown to be no longer symmetrical due to the motion of the upper tools.

In a second step, mechanical results were shown for a more complex geometry in which the pressure values are heterogeneous, causing quite different local densification kinetics. The preceding results show that the coupled electrical-thermal-mechanical finite element simulation may be of great help in understanding the temperature distribution and the densification during the SPS process. Such simulations should allow engineers to better determine the nature and geometry of the involved tooling materials, to better control microstructure formation. Forthcoming developments should aim at enriching the finite 
element model through the elimination of the different simplifying assumptions mentioned along the paper.

[1] R. Orru, R. Licheri, A. Mario Locci, A. Cincotti, G. Cao, Mater. Sci. Eng. R, 2009, 63, 127.

[2] J.R. Groza, ASM Handbook Powder Metallurgy, 1998, 7, 583.

[3] E. Olevsky, L. Froyen, Scripta Mater., 2006, 55, 77.

[4] E. Olevesly, S. Kandukuri, L. Froyen, Key. Eng. Mater., 2008, 368, 1580.

[5] U. Anselmi-Tamburini, S. Gennari, J.E. Garay, Z.A. Munir, Mater. Sci. Eng. A, 2005, $394,139$.

[6] X. Wang, S. R. Casolo, G. Xu, J.E. Garay, Acta Mater., 2007, 55, 3611.

[7] A. Zavaliangos, J. Zhang, M. Krammer, J.R. Groza, Mater. Sci. Eng. A, 2004, 379, 218.

[8] G. Molénat, L.Durand, J. Galy, A.Couret., J. of Metall., doi:10.1155/2010/145431.

[9] S. Shima, M. Oyane, Int. J. Mech. Sci., 1976, 18, 285.

[10] M. Abouaf, J.-L. Chenot, G. Raisson, P. Bauduin, Int. J. Num. Meth. Eng., 1988, 25, 191.

[11] R. Green, Int. J. Mech. Sci., 1972, 14, 215.

[12] M. Abouaf, J. L. Chenot, J. Theorical Appl. Mech., 1986, 5, 121.

[13] B. Julien, D. Hugues, T. Coupez, Int. J. Numer. Meth. Eng., 2009; 78, 980.

[14] C. Gruau, T. Coupez, Comput. Methods Appl. Mech. Eng., 2005, 194, 4951.

[15] T. Coupez, H. Digonnet, R. Ducloux, Appl. Math. Modelling, 2000, 25, 153.

[16] T. Nieh, L. Hsiung, J. Wadsworth, Intermetallics, 1999, 7, 163.

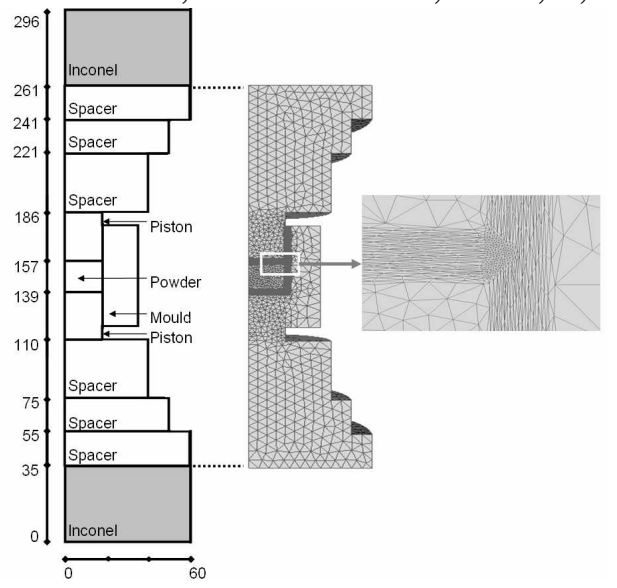

Fig. 1. Geometry of the SPS set-up with the generated mesh used in the simulation and a zoom in the region of the sample showing refined elements around the sample/matrix interface (radius of the sample $18 \mathrm{~mm}$, of the matrix $35 \mathrm{~mm}$ ).

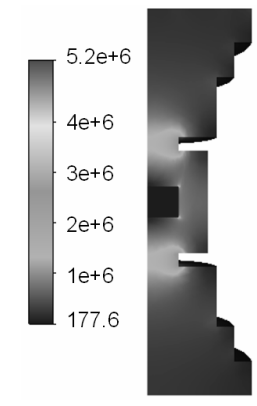

a)

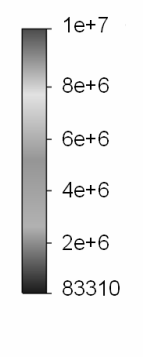

b)

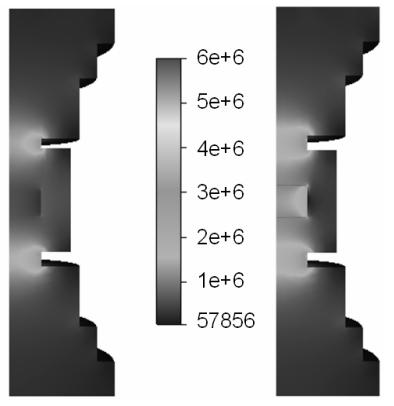

c)

Fig. 2.Distribution of the norm of the current density $\left(A / m^{2}\right)$ : a) Alumina sample after $\left.25 \mathrm{~s}, b\right)$ Alumina sample after $900 \mathrm{~s}, \mathrm{c}$ ) TiAl sample after $25 \mathrm{~s}$ 

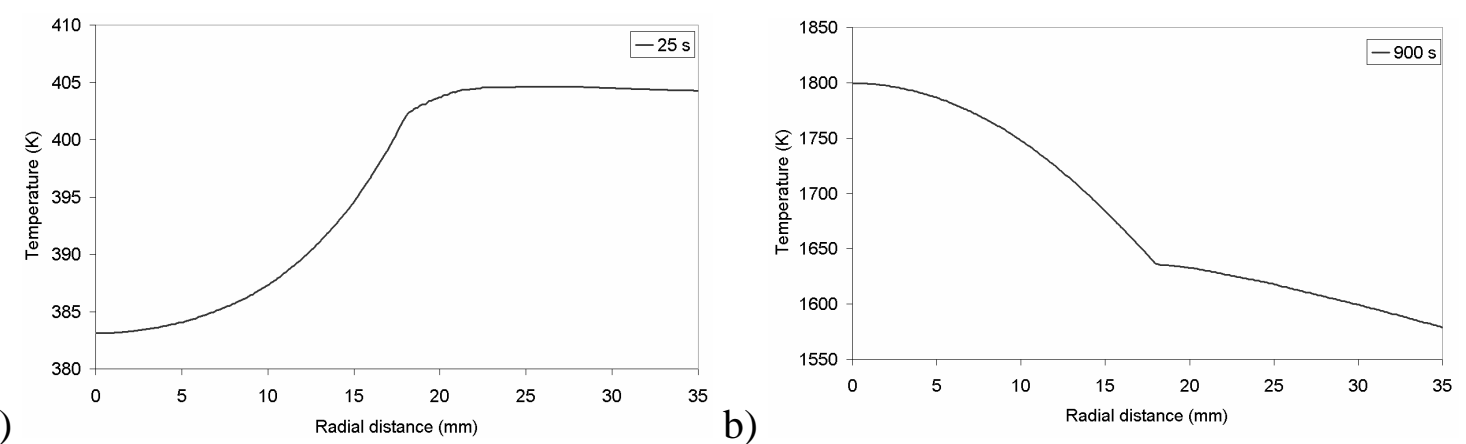

Fig. 3.Variation of the temperature along the radial axis from the center of the alumina specimen up to the edge of the die, a) $25 s, b) 900 s$.
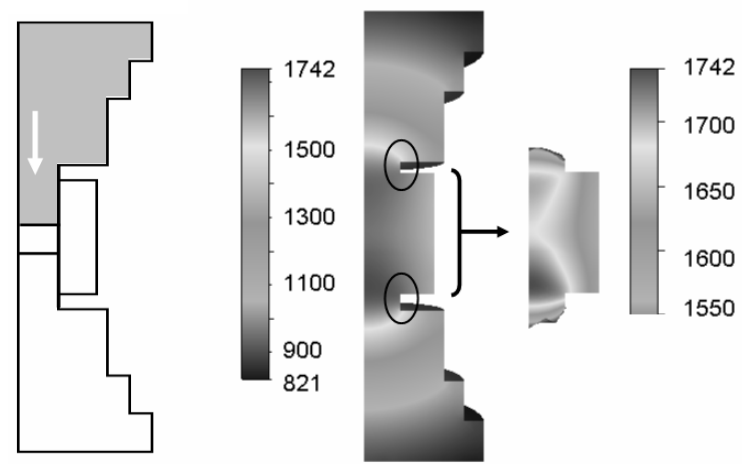

Fig. 4. Vertical motion of the upper tools causing non symmetrical distribution of the temperature in SPS machine. The extreme right picture better shows the effect by filtering temperature values in the central region.
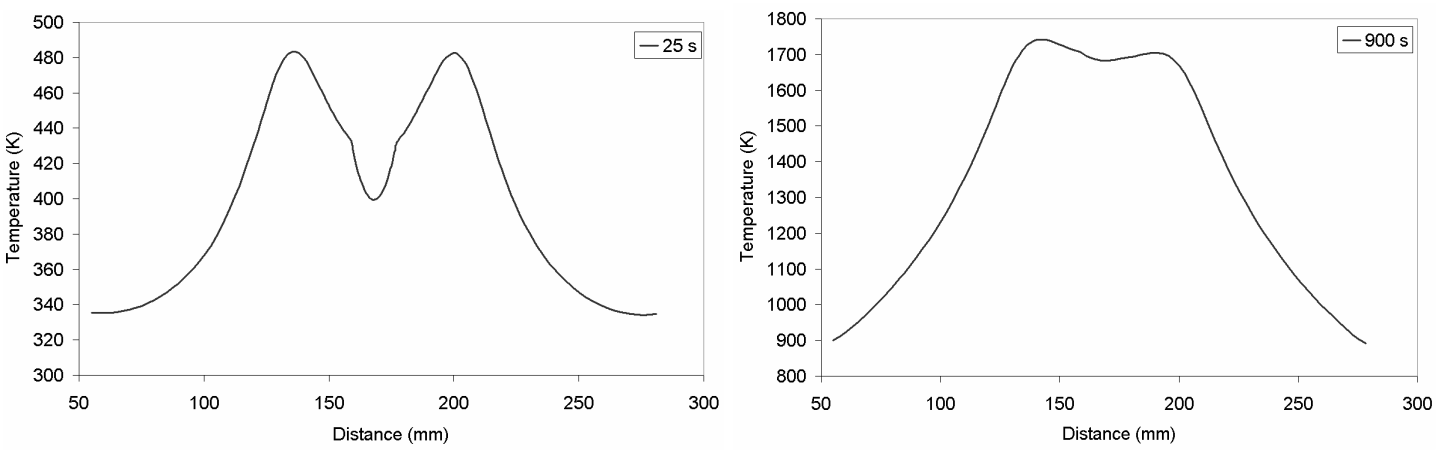

Fig. 5. Temperature variation along the vertical axis for the TiAl sample for different time steps showing the effect of the symmetry. 


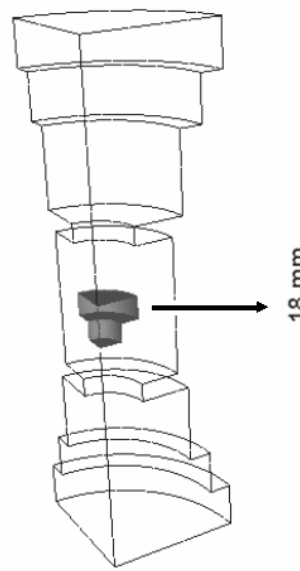

a)

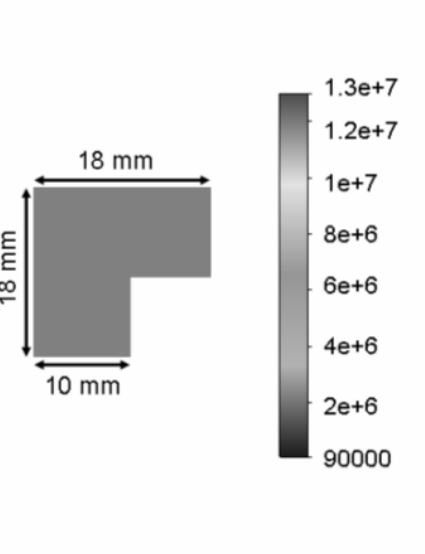

b)

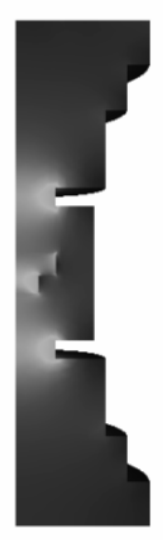

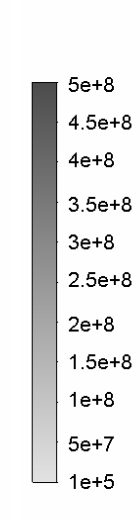

c)

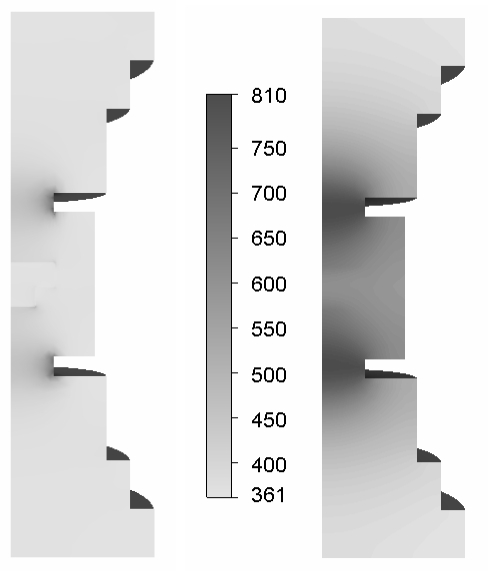

d)

Fig. 6. a) Shape and dimension of the initial powder domain; distribution of the norm of the current density (b), of the source term of heat equation (c) and of the temperature (d) after $25 \mathrm{~s}$.
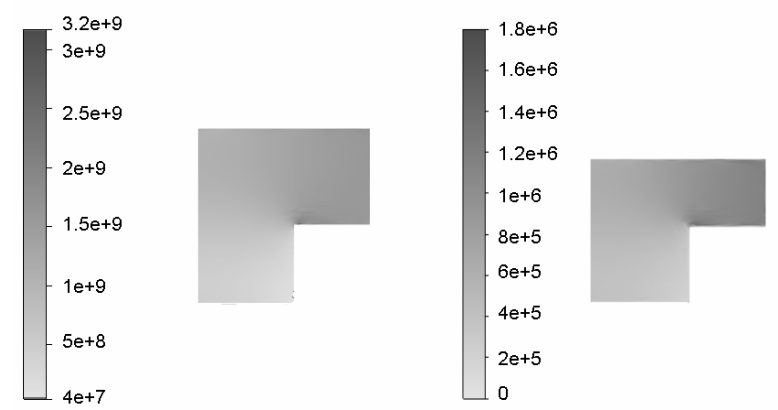

Fig. 7. Distribution of the pressure inside the TiAl powder at $25 \mathrm{~s}$ and after the height reduction at $925 \mathrm{~s}$.

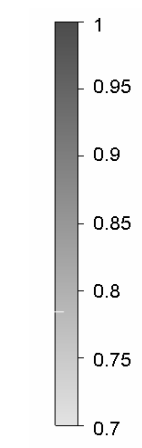

a)

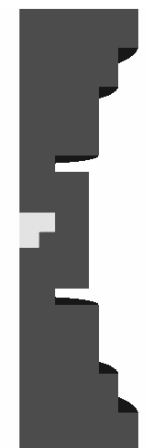

b)

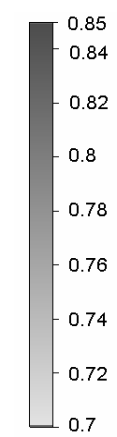

)

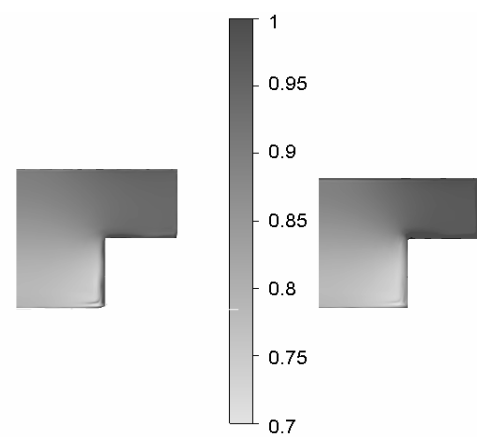

c)

Fig. 8.Initial relative density set to 0.7 in the TiAl powder and 1 in the tools (a) and its distribution in the sample after $425 s(b)$ and $925 s(c)$. 